\title{
PROTOTYPING IN SOCIAL VR: ANTICIPATE THE UNANTICIPATED OUTCOMES OF INTERACTIONS BETWEEN AI-POWERED SOLUTIONS AND USERS.
}

\author{
Mariani, Elena (2); \\ Kooijman, Finn Søren Casper (1); \\ Shah, Priyanka (1); \\ Stoimenova, Niya (1) \\ 1: Delft University of Technology; \\ 2: Politecnico di Milano
}

\begin{abstract}
Interactions of users with AI powered solutions (AIPS) have the potential to affect collective behaviours and amplify unanticipated outcomes. Product developers, organisations, and companies are increasingly being expected to take responsibility for the unanticipated outcomes of their products. In this paper we explore a proactive approach to prototyping AIPS-user interactions using Social Virtual Reality (SVR) environments that allows for the anticipation of potential outcomes. We contend that doing so would limit the detrimental effect outcomes could have on product developers' resources and reputation.
\end{abstract}

Keywords: Virtual reality, Virtual Prototyping, Artificial intelligence, Design process, Unanticipated outcomes

\section{Contact:}

Kooijman, Finn Søren Casper

Delft University of Technology

Industrial Design Engineering

Netherlands, The

kooijmanfinn@gmail.com

Cite this article: Mariani, E., Kooijman, F. S. C., Shah, P., Stoimenova, N. (2021) 'Prototyping in Social VR:

Anticipate the Unanticipated Outcomes of Interactions between AI-Powered Solutions and Users', in Proceedings of the International Conference on Engineering Design (ICED21), Gothenburg, Sweden, 16-20 August 2021. DOI:10.1017/ pds. 2021.510 


\section{INTRODUCTION}

AI-powered solutions (AIPS) are becoming exponentially embedded in consumer- and business-facing applications, even for critical purposes such as medical treatment, judicial evaluation, and financial analysis (Stoimenova \& Kleinsmann, 2020; Stoimenova \& Price, 2020). They are increasingly serving as an extension of the human mind, and consequently, are becoming well-positioned to create personalized human realities and shape human behaviour (Stoimenova \& Price, 2020; Rahwan, et al., 2019). More so, it could be expected that future implementations of AIPS take on physical manifestations, such as robots, in particular for products involving customer interaction, also leading the customers to form more personal bonds (Davenport et al., 2019).

Yet in the present day, despite the impressive progress, when left without any social evaluation (Maybury, 1990), these solutions can propagate biases in society, polarize opinions, manipulate our behaviour and affect our mental wellbeing (Guszcza, et al., 2018). Such undesired resultant effects are neither intended nor (arguably) anticipated by the creators of AIPSs. In order to be able to anticipate such failures, experiencing failures is necessary (Cook, 2002). Don Ihde (2008) affirms, "no matter the technology-medium, one can never fully design into a technology its purposes and uses". People will always behave in unintended ways. Further still, every technology, especially AI, has multiple uses and development trajectories, which cannot be fully inferred until it is put to use in a real environment (Stoimenova \& Price, 2020). One approach to anticipating these failures can be to prototype for product-user interactions in early stages of the AIPS development process. Prototypes are increasingly being used within industries to elicit requirements for complex problems (Jobst \& Meinel, 2013) by making the unknowns known (Jensen, et al., 2017). These prototypes can help industries to anticipate unanticipated outcomes and help designers to learn, discover, validate, or discard the decisions taken during the product development process (Ferrise, et al., 2013a; Stoimenova \& Kleinsmann, 2020). Prototyping in the early stages of the product development process has various benefits. They can minimize the occurrence of errors that may otherwise emerge later in the design process (Deininger, et al., 2017). As defined by Stoimenova \& Kleinsmann (2020), "prototypes should provide help in discovering new aspects of the problem at hand and support the invention of design requirements. As such, they can take any form, shape, and appearance based on [...] the phase of the design process in which they are being used". Assumptions about the desirability of product features and consequently the possible product user interactions could be tested for, without the development of the physical product. This would save time and resources, allow for quick iterations while limiting the undesired outcomes due to unanticipated interactions.

An effective way for product developers to prototype iteratively for product user interactions within short durations and at a low cost is to use Virtual Reality (VR) (Bruseberg \& McDonagh Philp, 2020; Füller \& Matzler, 2007; Ferrise, et al., 2013b). Recently, different VR environments have been increasingly adopted to run tests characterized by a higher degree of interactivity compared to traditional prototyping experiences, such as Immersive Service Prototyping (Razek, et al., 2018). VR is gaining traction in fields like surgical training, rehabilitation, STEM education, industrial design, architecture, real estate, etc. (Novak-Marcincin, et al., 2014; Cipresso, et al., 2018). Furthermore, VR is often used to test with users for accuracy, feasibility, desirability and aesthetic in the later stages of the product development process, when a sufficient amount of features and characteristics of the product have already been established. Several VR simulations are designed in environments that mimic the laws of the real world (e.g., gravity) rendering the training to be transferable to the real world, such as self-driving cars trained in the virtual world (Nascimento, et al., 2019). VR has also proven to be a valid method to study human interaction (real or virtual), and users' interaction with objects and environments, thanks to the immersion and the feeling of presence it can generate (Pan \& Hamilton, 2018; McVeigh-Schultz, et al., 2018). Recently, user interactions with working prototypes at the end of product development process are being analysed in VR to develop worthwhile experiences for products to be launched on the consumer market (e.g., Ferrise, et al., 2017; Lim, et al., 2008). We acknowledge that the use of VR platforms and Social VR $(S V R)$ platforms, by users and researchers globally, is increasing (Hackl, 2020; Jonas, et al., 2019). SVR platforms offer immersive environments and experiences for people to meet, work, and connect with each other while being in remote locations Given the advantages of utilising VR environments, this paper reports on a suggestion for an approach that enables prototyping during the conceptual phase of AIPS development so that unanticipated outcomes can be elicited by generating design hypotheses to be tested iteratively. Since the use of fully virtual prototypes is a cost and time effective solution, we 
developed and actuated our prototypes in a social VR environment (SVR). Based on these insights, our research proposes that SVR platforms, such as AltspaceVR, offer the potential to be an optimal environment to test and observe AIPS-users' interactions. In order to explore how an SVR platform can be used as a tool to identify and influence unanticipated and anticipated outcomes, we designed an interactive Virtual Prototype (iVP) (Ferrise, et al., 2013a) of an AI-powered virtual voice assistant (AIA). An iVP, as stated by Ferrise, et al. (2013), is a virtual prototype that "is used to test activities involving direct human interaction". This would help us anticipate the possible complexities without the cost and time it takes to prototype in real life. Through this approach we propose a method that allows us to develop an interactive prototype in the conceptual phases unlike other known prototyping approaches.

Therefore, the research question this article aims to answer is as follows: "How can we use prototyping in social VR to anticipate the unanticipated outcomes of AIPS-users' interactions?" Answering this question could help AIPS developers to reduce the emergence of unanticipated outcomes, resource consumption, and the cost of prototyping. The remaining paper will be structured as follows: the Method section will describe our case study and explain the rationale behind the choice of prototyping an AIA and the choice of the SVR platform. It will also discuss how we prototype AIAuser interactions to identify and influence unanticipated outcomes. Finally, we also describe the techniques we used in our research and the framework for the data analysis. The Results section presents the observations from the users' test and the iterative structure we adopted. This will be followed by the Discussion section, where we will point out the advantages of using the process of interactive prototypes we proposed. Finally, in the Conclusion, we refer to the stakeholders that might be able to benefit from the process adopted in our research.

\section{METHOD}

We adopted a Research through Design methodology (Giaccardi \& Stappers, 2017) and developed an escape room in AltspaceVR to stimulate an AIA-user interaction using the Wizard of $O z$ method. Participants were tasked to solve three clues in an escape-room game scenario guided by the AIPS. Escape-rooms are often designed to trigger interactions through a set of difficult tasks. Hence, the prototype setting was designed to implicitly require participants to ask for help and interact with the AIPS as they would in real-life scenarios. For example, a real life AIPS-user interaction was recreated, namely the need for searching information unknown to the user through Apple's Siri or Amazon's Alexa AIA's, by tasking the users to decode a morse code message not solvable without the help of an AIA. Below we explain our process in detail.

First, to elicit unintended outcomes and generate design hypotheses with participants, we needed to investigate the possibility to observe users' interaction in the early stages of the product development process. To do so, lo-fi interactive prototypes of AIPS products were needed. Furthermore, we needed a medium suitable for virtually simulating the interaction we sought without exposing participants to the real-life unintended outcomes that could have arose from their use of these products. We opted for SVR platforms because they combine the immersive benefits of Virtual Reality with an existing network of VR users. These immersive benefits in VR are characterized by two main aspects: the presence that is described as the sense of "being there" (Cipresso et al., 2018), and agency giving capability to maintain control over your actions in the virtual simulation (Winkler, et al., 2019). Moreover, SVR platforms offer the possibility to reach out and onboard a vast plethora of participants keen on actively participating and interacting.

In our investigation we simultaneously considered which SVR platform to use and which AIPS to prototype. The choice of the SVR platform affects the AIPS to be prototyped and vice versa. On one hand, we studied different SVR platforms, their characteristics and their suitability to the realization of interactive prototypes to be tested with users. On the other, we evaluated the AIPSs that would have been possible to reliably prototype on such SVR platforms.

We analysed a multitude of SVR platforms such as RecRoom, VR Chat, Mozilla Hub, Vtime XR, and AltspaceVR. To do so, we took a host of factors into account such as: desktop or mobile versions supported, availability and degree of complexity of embedded environment building tools, suitability for environment exploration, the possibility of haptic interaction with objects, supporting media streaming tools, degree of graphical realism, and degree of movements and interaction's realism. Therefore, the choice was driven by the availability of pre-designed elements a platform offers that can be customized without any prior knowledge on environment building tools (e.g., Unity game engine). 
The only platform that fulfilled the criteria demanded by the type of prototypes we sought to test is AltspaceVR. This is an SVR platform where people meet, attend shows, meetups, classes, or similar kinds of public events. It gives the possibility to share the same virtual space with others, and also to customize it by selecting assets from a library of structures, flora, interactives, and shapes that are created by AltspaceVR's team itself and by other users.

Simultaneously, the platform's choice directly influenced the selection of the AIPS to be tested, leading us to opt for an AIA. This choice was informed by three exploratory prototyping sessions, labelled as the $A$-Set, that we carried out to understand the platform. Here, the context, such as the affordances of the environment and the characteristics to be included in further prototyping sessions were investigated. As a result, we found that: first, while haptic interactions with objects were difficult to replicate, the platform has a good sound system, which allows for spatialized sound, and the ability to stream any kind of video footage to present information. Taking these affordances into consideration, we chose to prototype an AIA since the vast majority of people are already used to the interaction model it comes with, even if experienced in different use cases and at different degrees of complexity (e.g., voice searching in Chrome browser, Alexa, etc.). The simulated AIA, which mimicked the response patterns of a real one, was able to give general information about how to perform the tasks, carry out web searches and display the results on a big screen (as shown in Figure 1 and Figure 2).

Towards the end of the data gathering period, we categorized the gathered data taking inspiration from and further building upon a structure proposed by Stoimenova and Kleismann (2020). The primary categories proposed by Stoimenova and Kleismann (2020) were Purpose, Frame, and Mode of action.

The Purpose is what is intended during the experiment. The Frame is a result of Purpose and Data combined, where the Data is gathered from literature, observed outcomes emerged in previous experiments (both desired and undesired), and from the insights collected during the interviews with the participant at the end of each prototyping session. The Mode of Action is how the product responds to the external environmental influences and as a result tries to influence the environment. The Prototype is generated by Frame and Mode of action. More precisely, given the iVPs' focus on testing while involving direct human interaction, every version of our iVP will result from the combination of Prototype and Actuation. Here, the Actuation is the individual interaction of the user with the prototype. This interaction results in generation of values, intended or unintended. We expanded the term unintended values in three further categories as Desired Anticipated Outcomes (DAO), Undesired Anticipated Outcomes (UAO), Influenced Anticipated Outcome (IAO).

Therefore, every prototyping session comes with its own set of anticipated outcomes, which generates iteratively the design hypothesis for the following prototyping session. Only by making these explicit, the identification of new unanticipated ones becomes possible. By eliciting unanticipated outcomes, we can then identify if these are desirable or not. An outcome was considered desirable if it made the AIA-user's interaction more pleasant, flawless, and hence able to add positive value to the interplay required to successfully perform the tasks. Hence, if they have a positive value for the user they will be considered as DAOs for the coming prototype sessions. Nevertheless, if they do not provide positive value, and their occurrence should be actively avoided, they will be recognised as IAOs, and addressed in the coming session by actively influencing and modifying specific characteristics of the prototype setting. It is important to note that what makes an outcome desirable or not are values that the participant is not necessarily aware of. It means that DAOs and UAOs could emerge from a user's feedback during the final interview, as well from difficulties experienced while executing tasks and noted down during the session's observation activity.

More concretely, the outcomes elicited from the A-set formed the basis of the first B-Set prototyping session setup and its set of anticipated outcomes regarding the participants' interaction with the product in the context. Through an additional ten iterative prototyping sessions, labelled as the B-Set, unanticipated outcomes, with respect to product-user interaction, product characteristics, and circumstantial occurrences were identified and, if not desirable, actively influenced. During the B-Set prototyping sessions, each lasting 15-45 minutes, ten members of the AltspaceVR community were invited to participate. To onboard and convey information about the prototype sessions we created and used public events on AltspaceVR, and the AltspaceVR's Discord channel (an external instant messaging platform). Here, participants were briefed about the escape-room scenario, the kind of support they could seek from the AIA, and the nature of questions they could ask.

The context and environment of the escape-room evolved during the prototypes and the main stimuli presented to the subject were visual and auditory. The primary source of the visual stimuli was 
a big screen, showing a browser and the results of web searches. This allowed us to simulate how an AIA would provide its findings to its user in real life. Furthermore, minor visual clues were also distributed on the escape-room's walls (i.e., posters) (Figure 2). Auditory stimuli were generated using the Wizard of $\mathrm{Oz}$ method. The form and manifestation (scale, dimensions, appearance) of the AIA was close to existing ones such as Alexa and Google home. The riddles we used were designed in such a way that the participants would need the help of the AIA. For instance, we asked our participants to solve morse code messages, find the coordinate location of a landmark, and solve a rebus.

While the participants were solving the tasks, we carefully recorded our observations in descriptive notes concerning the participants' decisions on how to approach the task, when and how they sought help from the AIA, and what language they used to address the AIA (e.g., formal, or informal). Furthermore, every session was video recorded in order to be analysed later on and to identify the context in which a given unanticipated outcome emerged.

Upon completion of the escape-room, we carried out 5-15 minute semi-structured interview with each participant. Ultimately, we used participant validation, through member checks (Ravitch \& Mittenfeller N, 2015), to have the participants reflect on their experience and perception of the AIA's fidelity level and to verify the level of immersion of the iVP.

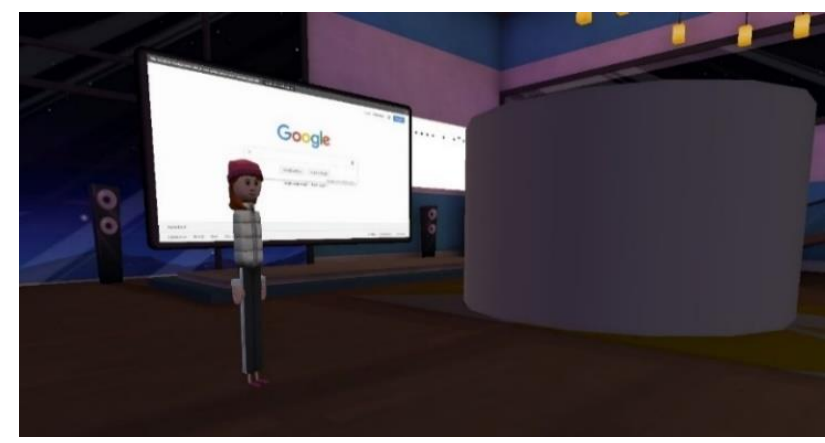

Figure 1. A-Set, session 3: Created using a template SVR environment. The AIA manifestation is an out-of-scale cylinder in the middle of the room.

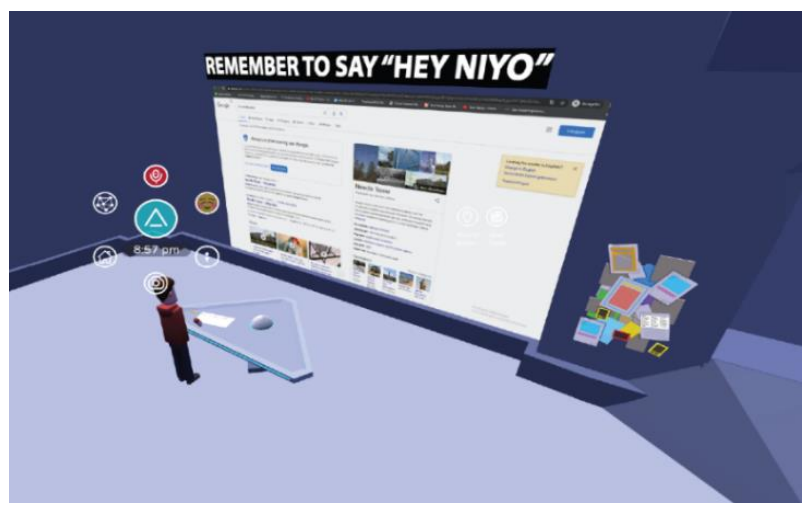

Figure 2. B-Set, session 9: The participant is performing a web research to solve a clue while interacting with the AIA in the custom-built escape room environment.

\section{RESULTS}

The results presented below show the outcomes of the A-Set and B-Set prototypes. Furthermore, it shows the iteration process and how consecutive experiments influenced each other.

\subsection{A-Set prototypes: understanding how to prototype in VR}

We carried out three prototyping sessions, each with its unique focus. The first allowed us to gain insight into the applicability of qualitative research methods in SVR. The prototype was carried out in a template virtual environment, modelled after a rooftop terrace (Figure 1). One of the discoveries made during this prototype session was that qualitative research methods such as member checks could be implemented. The second exploratory prototype provided insights into the ability of the platform to create feelreal contexts (Vinuesa, et al., 2020) and preserve the environment's complexity. We started with using 
a pre-existing environment and modified it by adding template assets on the SVR platform which allowed us to test the platform's ability to simulate fictional and non-fictional environments. This was perceived by users as immersive experiences thanks to the VR's characteristics of presence and agency. Furthermore, the ability to integrate presenter screens and a web browser was a key discovery during this exploratory prototype, as it further allowed us to mimic the way AIAs deliver information. Lastly, it is important to note that it was not possible to receive haptic feedback from interaction with objects.

The third exploratory prototype was aimed at understanding the users' level of engagement through the sought interaction and acceptance of the proposed setting. It demonstrated that increased fidelity prompted further engagement and acceptance of the created virtual environment. Moreover, it was noted that novel interactions and experiences increased people's interest and willingness to participate in the prototype sessions. This was another reason why we adopted an escape-room scenario, as it is naturally engaging and immersive in the context.

The outcomes of these A-Set prototypes shaped our understanding of the SVR environment, the potential, and its limitations. Upon completion of the A-Set, we were able to have a set of Desirable Anticipated Outcomes (DAOs) made explicit and observe and note the emergence of unanticipated outcomes during each prototyping session of the following B-Set.

\subsection{B-Set prototypes: understanding how to elicit unanticipated outcomes in VR}

During the B-Set prototypes, we adopted an iterative process presented in Figure 3.

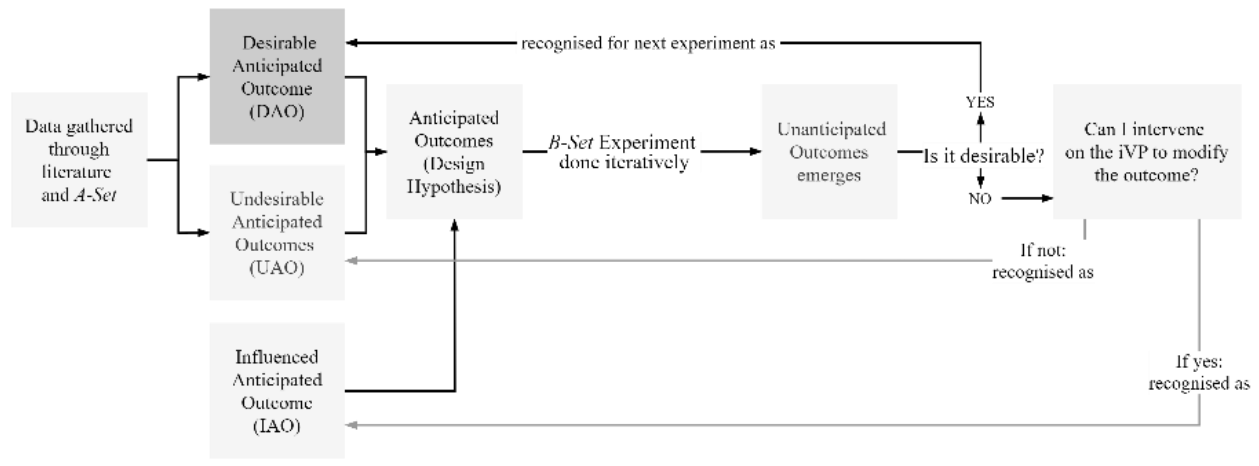

Figure 3. Iterative process adopted for B-Set prototyping sessions

Through the data gathered from literature and the A-set prototypes, a list of desirable anticipated outcomes (DAO) and undesirables anticipated outcomes (UAO) were generated. The DAO's and UAO's acted as the starting point for- and were expected to manifest in the prototype session and hence formed the design hypothesis for this prototype session.

Upon completion of each prototyping session, the unanticipated outcomes were analysed on desirability. If the outcome was desirable, the unanticipated outcome was recognised as a DAO for the next experiment. However, an additional step was required if the unanticipated outcome was undesirable. If it was possible to intervene on the iVP to trigger a different outcome, the experiment setup was actively influenced to eliminate the unanticipated outcome from occurring again. This change to the experiment setup usually implied modifications of the AIA manifestation and/or the interaction script and/or visual stimuli presented in the prototype environment. As a result, this unanticipated outcome was recognised as an Influenced Anticipated Outcome (IAO) for the next experiment. Nevertheless, if it was not possible to intervene on the iVP, the unanticipated outcome was recognised as an undesirable anticipated outcome for the next experiment.

To illustrate the process described above, let's take the transition we made between prototyping session $2 \mathrm{~B}$ and $3 \mathrm{~B}$ as an example. It was characterised by the following unanticipated outcome: "participants did not perceive voice as a manifestation of the AIA". This occurred because the physical manifestation of the AIA was not convincing, due to its scale (see Figure 1), and because scripted interaction between the AIA and the participant was lacking. In order to actively influence these unanticipated outcomes, two aspects were altered during prototype session 3B. Firstly, the manifestation of the AIA was made to resemble what already exists in the AIA product portfolio, namely Google home or Amazon's Alexa (see the spherical object on the table in Figure 2). Secondly, the interaction script 
was modified by adding standard scripted responses based on the standard response pattern of the Google Assistant. Through these scripted responses, the interactions between the participant and the simulated AIA became more similar to conventional AIA's, improving the product-user interactions and therefore the believability of the Wizard of Oz. Then, due to this modification of the prototype session setup and the consequent improvement of the product-user interaction, we listed "Participants will perceive voice as a manifestation of the AIA" as IAO.

To illustrate the process that generated UAOs, session 8 of the B-Set will be taken as an example. The VR platform caused participants to glitch out of the VR environment during the session. Due to the technical nature of this unanticipated outcome, it could not be resolved by actively influencing the upcoming iVP. Hence, it could only be anticipated to happen in the following ones.

Table 1 illustrates how the data was clustered, using the structure inspired from Stoimenova and Kleismann (2020), during the last experiment, namely the 10th from the B-Set.

Table 1. Data collected during 10th prototyping session of B-Set.

\begin{tabular}{|l|l|}
\hline B-Set: Prototyping session 10 \\
\hline Purpose & $\begin{array}{l}\text { Wizard of Oz AIA aiding the participant to solve the clues. } \\
\text { If people need help in the escape-room they will interact with a stationary } \\
\text { through scripted interactions and standard responses, and only responds } \\
\text { when a start word/code is used. }\end{array}$ \\
\hline Mode of action & $\begin{array}{l}\text { The AIA provides assistance through a completely scripted interaction and } \\
\text { standard responses. }\end{array}$ \\
\hline $\begin{array}{l}\text { Desired Anticipated } \\
\text { Outcomes }\end{array}$ & $\begin{array}{l}\text { Participants will use Hey Niya for activating the AIA } \\
\text { Participants will interact with the AIA. }\end{array}$ \\
\hline $\begin{array}{l}\text { Undesired Anticipated } \\
\text { Outcomes }\end{array}$ & $\begin{array}{l}\text { Participants might use resources outside of the resources within the virtual } \\
\text { environment (the AIA). }\end{array}$ \\
\hline $\begin{array}{l}\text { Unanticipated } \\
\text { outcomes }\end{array}$ & $\begin{array}{l}\text { Participants did not perceive the voice as an AIA due to the discrepancy in } \\
\text { the AIA's name and the search engine used to display information. }\end{array}$ \\
\hline iVP changes & $\begin{array}{l}\text { Script was altered to provide additional details on how to solve the clues, } \\
\text { as the participant in session 9 was unable to successfully complete the } \\
\text { clues. }\end{array}$ \\
\hline $\begin{array}{l}\text { Influenced Anticipated } \\
\text { Outcomes }\end{array}$ & Participants will perceive voice as a manifestation of AIA. \\
\hline
\end{tabular}

Through this process, a clear relationship started to emerge between the decrease of unanticipated outcomes, the increase of DAOs and UAOs, due to actively influenced IAOs. As shown in Figure 4 during the length of the B-set, by actively influencing the IAOs and identifying the UAOs and the DAOs, it became possible to create a setup that reduced the emergence of unanticipated outcomes.

\section{DISCUSSION}

Prototypes are recognized as useful tools for testing product functionality, desirability, performance and at times observing and analysing the user's interaction with the product. Hence, they are generally aimed at confirming or disconfirming a given hypothesis the design team has. The process we have shown in this paper could aid designers in generating hypotheses regarding product-user interactions in the early stages of the process by iteratively making assumptions and insights explicit through prototyping. This will result in the possibility for the design team to generate a set of design hypotheses and elicit unanticipated outcomes to prevent them from occurring after product commercialisation.

Each prototyping session was characterized by a different set of design hypotheses, which originated from the established Desired and Undesired Anticipated Outcomes (DAOs and UAOs), the actively Influenced Anticipated Outcomes (IAO) and the corresponding modifications made to the iVP (AIA manifestation and/or the interaction script and/or visual stimuli presented in the prototype environment). The evaluation of the outcomes generated by our intervention confirmed or disconfirmed the current design hypothesis. Meanwhile, it also allowed us to further produce new design hypotheses, triggering an iterative alteration of the iVP characteristics. When a certain level of saturation is achieved, which occurs when the emergence of unanticipated outcomes decreases and the amount of DAOs emerging 
increases, a manifestation of how the product-user interaction would be like in the designed environment can be created. These can then be worked with in further stages of the product development process.

The graph below is a visualization of changes in the observed number of unanticipated outcomes, Influenced Anticipated Outcomes (IAOs), Undesired Anticipated outcomes (UAOs), and Desired Anticipated Outcomes (DAOs) over the course of the B-Set prototyping sessions.

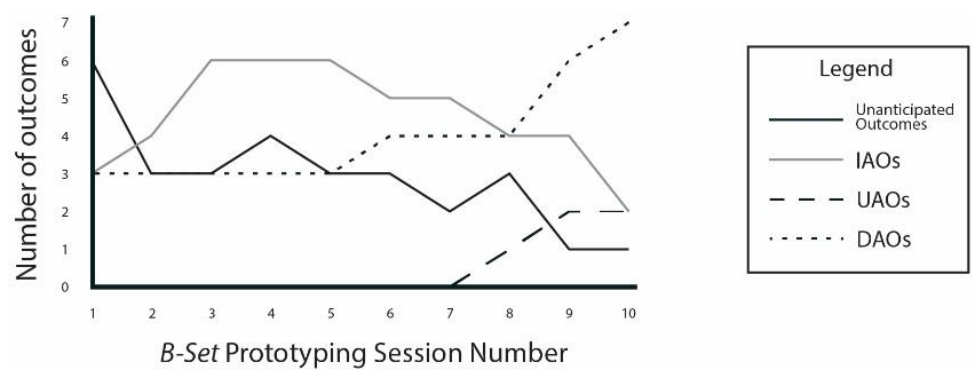

\section{Figure 4. Visualisation of the trend in the unanticipated outcomes, Desired Anticipated Outcomes (DAOs), Undesired Anticipated Outcomes (UAOs) and Influenced Anticipated Outcomes (IAOs).}

Understanding the desired and undesired product-user interactions in the early stages of the product development cycle is of paramount importance for organizations. Doing so would allow them to avoid successive resource-intensive modifications that might harm their reputation (Bjoerkli, 2015). The Wizard of Oz prototype technique proved to be applicable because it could allow the researcher to simulate how the product-user interaction should look like prior to the thorough development of the product features. This recommendation is also valid for the AIPS developers and producers especially due to the complexity of the interaction of their product with a wide number of users. In fact, the operational opacity of AIs makes it difficult to comprehensively understand its working and often produces the propagation of biases among its users (Stoimenova \& Price, 2020). This approach could enable the developers to early identify and proactively iterate the desired and undesired product-user interactions thus limiting the detrimental effect of unanticipated outcomes on AIPS developers' resources and reputation. Finally, the intrinsic characteristics of SVR platforms such as AltspaceVR open up innovative applications for the proposed prototyping process. SVR is a free tool with a global reach and its use increased exponentially during the COVID-19 outbreak (Duboff, 2020). Most of the users of the SVR platforms are already familiar with VR environments and possess the necessary VR literacy. Hence, they do not need to be instructed about the allowances and limitations of the environment (Gunkel, et al., 2019). Nevertheless, it is relevant to notice that participants' nonverbal responses were limited. In the AltspaceVR environment, body movement and facial expression are bound to basic head and hand movements if the participant is using an HMD (Head Mounted Device) and controllers. This resulted in a limited ability to obtain additional insights from nonverbal cues. We also observed that if the prototype setting is engaging, such as the escape-room scenario used during this research, participants are disposed to interact and collaborate without the need for compensation. It is important to notice how SVR is characterized by a high degree of anonymity because users are free to choose pseudonyms and freely customize their avatar appearance (Kohler, et al., 2009). This might be beneficial as it gives the possibility to users to better express their "true self" in comparison to a face-to-face setting. Moreover, in such environments lead users with a technical understanding of emerging technologies are easy to find, also supported by Fuller, et al. (2004). Their contribution can be integrated into the hypothesis generation process of the design team (Füller \& Matzler, 2007); hence, we recommend AIPS developers and producers to integrate the use of SVR platforms early in the prototyping process to generate hypotheses about product-user interactions. Simultaneously, we recommend SVR platform developers or producers to improve the existing infrastructure to accommodate this growing need while working closely with AIPS developers and researchers studying user-product interactions. As a scope for further research, a range of AIPSs can be tested in SVR in order to confirm the applicability of the process and to build a theory from it.

\section{CONCLUSION}

The world is rapidly evolving and the growing integration of AI in our daily lives seems inevitable. AIPSs have been shown to produce many unanticipated outcomes as they have a tendency to propagate biases in society, polarize opinions, spread misinformation, manipulate our behaviour and 
affect our mental wellbeing (Guszcza, et al., 2018). Hence there is an urgent need for effective methods that support hypothesizing the potential unanticipated outcomes during the development phase of AIPS. Prototyping usually takes place in the late stages of the product development process to validate product features. Hence, these methods are often reactive, rather than proactive, meaning that these unanticipated outcomes often still have an impact on society before being solved.

Hence, it is time for AIPS producers to take responsibility by exploring unanticipated outcomes through the proposed method of prototyping AIPSs in SVR environments. It also lends an opportunity for SVR platforms to tailor their tools and environments in order to make them more suitable for the purpose of research. The method proposed in this paper enables us to formulate design hypotheses iteratively while prototyping in a feel-real context without the risks of changing the real context or losing out on the complexity as in a controlled experimental setting (Vinuesa, et al., 2020). Due to the iterative nature of the process, unanticipated outcomes are repeatedly identified and resolved. Furthermore, we can nonintrusively observe the participant's natural behaviour which produces more accurate insights. Nevertheless, participant observation and interviews are limiting with regards to body language and facial expressions. Depending on the tested attributes, this might limit the richness of the collected data. In time, larger and more geographically diversified user groups could be reached by prototyping in SVR environments. Furthermore, the costs of prototyping in VR environments are lesser than in traditional ways, as test environments, objects, and scenery can be created and recreated digitally. With this research, we conclude that AIPS developers could use existing SVR platforms to re-evaluate their prototyping practices so that they can render out unanticipated outcomes in order to contribute to sustainable societal wellbeing. SVR platforms could see this as an opportunity and build in necessary tools to support the AIPS developers to conduct research in SVR. Finally, we acknowledge that the exploration of VR as a tool for research, although seemingly promising, is in a preliminary stage. Even less research is done for prototyping AI within SVR environments.

\section{REFERENCES}

Bjoerkli, L. E., 2015, “A Review of Virtual Prototyping Approaches for User Testing of Design Solutions”.

Bruseberg, A. \& McDonagh-Philp, D., 2020, "Focus groups to support the industrial/product designer: a review based on current literature and designers' feedback", Applied Ergonomics, Vol. 33 No. 1, pp. 27-38.

Cipresso, P., Giglioli, A. C. I., Raya, A. M. \& Riva, G., 2018, “The Past, Present, and Future of Virtual and Augmented Reality Research: A Network and Cluster Analysis of the Literature", Frontiers in Psychology. https://doi.org/10.1016/S0003-6870(01)00053-9.

Cook, R., 2002, "How complex systems fail", [online] Available at: https://www.researchgate.net/publication/ 228797158_How_complex_systems_fail (accessed 7 December 2020).

Davenport, T., Guha, A., Grewal, D., \& Bressgott, T., 2019, "How artificial intelligence will change the future of marketing”, Journal of the Academy of Marketing Science, Vol. 48, pp. 24-42. https://dx.doi.org/10.1007/s11747-019-00696-0

Deininger, M., Daly, R. S., Sienko, H. K. \& Lee, C. J., 2017, "Novice designers' use of prototypes in engineering design", Design Studies, Volume 51, pp. 25-65. https://doi.org/10.1016/j.destud.2017.04.002.

Duboff, R., 2020, "Virtual reality in the time of COVID-19", [online] Accenture. Available at: (https://www.accenture.com/us-en/blogs/interactive-insights-blog/virtual-reality-in-the-time-of-covid-19) (accessed 7 December 2020).

Ferrise, F., Bordegoni, M. \& Cugini, U., 2013, "Interactive Virtual Prototypes for Testing the Interaction with new Products", Computer-Aided Design and Applications, Vol. 10 No.3, pp. 515-525. http://doi.org/10.3722/cadaps.2013.461-474.

Ferrise, F., Bordegoni, M. \& Graziosi, S., 2013b, “A Method for Designing Users’ Experience with Industrial Products based on a Multimodal Environment and Mixed Prototypes", Computer-Aided Design and Applications, Vol. 10 No. 3, pp. 461-474. http://10.3722/cadaps.2013.515-525.

Ferrise, F., Graziosi, S. \& Bordegoni, M., 2017, "Prototyping strategies for multisensory product experience engineering", Journal of Intelligent Manufacturing volume, No. 28, p. 1695-1707. https://doi.org/10.1007/s10845-015-1163-0.

Füller, J., Bartl, M., Ernst, H. \& Muhlbacher, H., 2004, “Community based innovation: a method to utilize the innovative potential of online communities", 37th Annual Hawaii International Conference on System Sciences, Big Island, 2004. http://doi.org/10.1109/HICSS.2004.1265464.

Füller, J. \& Matzler, K., 2007, "Virtual product experience and customer participation - A chance for customercentred, really new products", Technovation, Vol. 27 No. 6, pp. 378-387. https://doi.org/10.1016/j.technovation.2006.09.005.

Giaccardi, E. \& Stappers, J. P., 2017, "Research through Design”, In: Interaction Design Foundation, The Encyclopedia of Human-Computer Interaction, 2nd Ed. 
Gunkel, N. B. S., Stokking, H., De Koninck, T. \& Niamut, O., 2019, "Everyday photo-realistic social VR: communicate and collaborate with an enhanced co-presence and immersion", International Broadcast Conference (IBC 2019) Amsterdam, 2019. https://doi.org/10.5281/zenodo.4040503.

Guszcza, J. et al., 2018, Why we need to audit algorithms. [online] Harvard Business School. Available at: https://hbr.org/2018/11/why-we-need-to-audit-algorithmsv (accessed 5 December 2020).

Hackl, C., 2020, Social VR, Facebook Horizon And The Future Of Social Media Marketing. [online] Forbes. Available at: https://www.forbes.com/sites/cathyhackl/2020/08/30/social-vr-facebook-horizon--the-futureof-social-media-marketing/?sh=31f479ca5b19 (accessed 7 December 2020).

Ihde, D., 2008, “The Designer Fallacy and Technological Imagination”, Philosophy and Design, pp. 51-59. https://dx.doi.org/10.1007/978-1-4020-6591-0_4

Jensen, B. M., Elverum, W. C. \& Steinert, M., 2017, "Eliciting unknown unknowns with prototypes: Introducing prototrials and prototrial-driven cultures", Design Studies, Vol. 49, pp. 1-31. https://doi.org/10.1016/j.destud.2016.12.002.

Jobst, B. \& Meinel, C., 2013, "How Prototyping Helps to Solve Wicked Problems", In: H. Plattner, C. Meinel \& L. Leifer, (Ed.), Design Thinking Research, Springer, pp. 105-113. https://dx.doi.org/10.1007/978-3-31901303-9.

Jonas, M., Said, S., Yu, D., Aiello, C., Furlo, N., \& Zytko, D., 2019, “Towards a Taxonomy of Social VR Application Design", (CHI PLAY'19 Extended Abstracts) Association for Computing Machinery, pp. 437-444). New York, NY, USA,: Extended Abstracts of the Annual Symposium on Computer-Human Interaction in Play Companion Extended Abstracts. https://dx.doi.org/10.1145/3341215.3356271

Kohler, T., Matzler, K. \& Füller, J., 2009, “Avatar-based innovation: Using virtual worlds for real-world innovation”, Technovation, Vol. 29 No. 6-7, pp. 395-407. https://doi.org/10.1016/j.technovation.2008.11.004.

Lim, Y.-K., Stolterman, E. \& Tenenberg, J., 2008, “The anatomy of prototypes”, ACM Transactions on Computer-Human Interaction, Vol. 15 No. 2, pp. 1-27. https://doi.org/10.1145/1375761.1375762.

Maybury, T. M., 1990, "The mind matters: artificial intelligence and its societal implications", IEEE Technology and Society Magazine, Vol. 9 No. 2, pp. 7-15. https://doi.org/10.1109/44.56350.

McVeigh-Schultz, J. et al., 2018, "Immersive Design Fiction: Using VR to Prototype Speculative Interfaces and Interaction Rituals within a Virtual Storyworld”, DIS '18, 2018, Association for Computing Machinery, p. 817-829. https://doi.org/10.1145/3196709.3196793

Nascimento, M. A. et al., 2019, “The Role of Virtual Reality in Autonomous Vehicles' Safety”, IEEE International Conference on Artificial Intelligence and Virtual Reality (AIVR), San Diego, 2019, IEEE, pp. 50-507. http://doi.og/10.1109/AIVR46125.2019.00017.

Novak-Marcincin, J., Janak, M., Barna, J. \& Novakova-Marcincinova, L., 2014, “Application of Virtual and Augmented Reality Technology in Education of Manufacturing Engineers", New Perspectives in Information Systems and Technologies, Springer, Cham, pp. 439-446. https://doi.org/10.1007/978-3-31905948-8_42.

Pan, X. \& Hamilton, F. d. C. A., 2018, "Why and how to use virtual reality to study human social interaction: The challenges of exploring a new research landscape", British Journal of Psychology, Vol. 109 No.3, pp. 395-417. https://doi.org/10.1111/bjop.12290.

Rahwan, I. et al., 2019, "Machine Behaviour”, Nature, Vol. 568, pp. 477-486. doi.org/10.1038/s41586-0191138-y.

Ravitch, M. S. \& Mittenfeller N, C., 2015, "Validity: Processes, strategies and considerations”, In: SAGE, Qualitative research: Bridging the conceptual, theoretical, and methodologica, pp. 185-213.

Razek, A. R. M. A., van Husen, C., Pallot, M. \& Richir, S., 2018, “A Comparative Study on Conventional versus Immersive Service Prototyping (VR, AR, MR)", The Virtual Reality International Conference - Laval Virtual, 2018. https://doi.org/10.1145/3234253.3234296.

Stoimenova, N. \& Kleinsmann, M., 2020, "Identifying and addressing unintended values when designing (with) Artificial Intelligence", Synergy - DRS International Conference 2020. https://doi.org/10.21606/drs.2020.222.

Stoimenova, N. \& Price, R., 2020, "Exploring the Nuances of Designing (with/for) Artificial Intelligence", Design Issues, Vol. 36 No. 4, pp. 45-55. https://doi.org/10.1162/desi_a_00613.

Vinuesa, R. et al., 2020, "The role of artificial intelligence in achieving the Sustainable Development Goals", Nature Communications, Vol. 11 No. 233. https://doi.org/10.1038/s41467-019-14108-y.

Winkler, P., Stiens, P., Rauh, N., Franke, T., \& Krems, J., 2019, "How latency, action modality and display modality influence the sense of agency: a virtual reality study", Virtual Reality Vol. 24, pp. 411-422. https://dx.doi.org/10.1007/s10055-019-00403-y. 